\title{
Multi-Wavelength Photoplethysmography Device for the Measurement of Pulse Transit Time in the Skin Microvasculature
}

\author{
Jukka-Pekka Sirkiä, Tuukka Panula, Matti Kaisti \\ University of Turku, Department of Future Technologies, Turku, Finland
}

\begin{abstract}
The purpose of this study was to develop a multiwavelength photoplethysmography (MWPPG) device for studying the skin microvasculature. Microvasculature, in general, is an important part of the cardiovascular system because of its role in microcirculation, which manages tissue nutrient and oxygen supply, and where much of the peripheral resistance occurs. The developed device utilises the fact that the penetration depth of light into the skin is depended on the light wavelength. Thus, an MWPPG device utilising multiple different wavelengths of light allows the studying of blood vessels at different depths. The device proved to produce good quality signal making it possible to compute the small time differences between the pulses measured at different wavelengths, and hence enabling the computation of pulse transit times in the skin microvasculature $\left(P T T_{M}\right)$. The order of the computed $P T T_{M}$ values are in line with the theory of how deep each wavelength of light penetrates into the skin. The results could be used in hemodynamic monitoring.
\end{abstract}

\section{Introduction}

Photoplethysmography (PPG) is an optical technique for measuring blood volume changes within the skin tissue by illuminating the skin with a light source. The changes in blood volume cause fluctuations in the amount of light detected by a photodetector located next to the light source, generating a pulse waveform which follows the cardiac cycle. PPG is the technique used in pulse oximeters, which are widely used in clinical applications for measuring blood oxygen saturation and heart rate. The pulse waveform generated by a PPG device can be used to compute pulse transit time (PTT), which is the time it takes for the pulse wave to travel from one arterial site to another. PTT is usually used to compute pulse wave velocity (PWV), which can be computed easily by dividing the distance between the two measurement sites by the PTT. PWV is considered as the gold standard in measuring arterial stiffness ([1]) and it is also related to blood pressure
([2]). Typically, PTT is measured from two large arteries, for example, carotid and femoral arteries, as in [1]. However, in [3], PTT in the skin microvasculature (denoted here by $\mathrm{PTT}_{\mathrm{M}}$ ) was computed using signals generated by a multi-wavelength PPG (MWPPG) device, and recently in [4], PTT $_{M}$ was related to blood pressure.

The working principle of applying MWPPG to skin microvasculature is based on the relation of light wavelength and skin penetration depth, discussed for example in $[5,6]$. In general, longer wavelength light penetrates deeper into the skin than shorter wavelength light when the wavelength is in the visible and in the beginning of the nearinfrared part of the spectrum. More specifically, blue and green light penetrate approximately just below epidermis (the outermost layer of the skin) where capillaries are located, yellow light penetrates approximately into arterioles in dermis (the second layer of the skin), and red and infrared light penetrate even deeper, approximately into arteries. The developed device uses these five wavelengths to study the different blood vessels with the goal of assessing microvasculature. In a previous article, [7], our research group presented a device capable of measuring blood pressure accurately from the fingertip, and the device presented here is a continuum to our effort to assess cardiovascular health from the tip of a finger in a non-invasive way.

\section{Methods}

\subsection{Hardware Description}

The hardware consists of two custom-designed printed circuit boards (PCB): a sensor PCB and an accompanying controlling PCB. The sensor PCB consists of five lightemitting diodes (LED) and a photodiode. The LEDs emit light at five different wavelengths: $465 \mathrm{~nm}$ (blue), 515 $\mathrm{nm}$ (green), $590 \mathrm{~nm}$ (yellow), $640 \mathrm{~nm}$ (red), and $880 \mathrm{~nm}$ (infrared). The wide range of wavelengths included requires a photodiode with a wide spectral bandwidth, which is why Vishay Semiconductor's VEMD1060X01 was selected. The photodiode has a spectral bandwidth of 350$1,070 \mathrm{~nm}$, peaking at $820 \mathrm{~nm}$.

The sensor PCB is connected to the controlling PCB via 


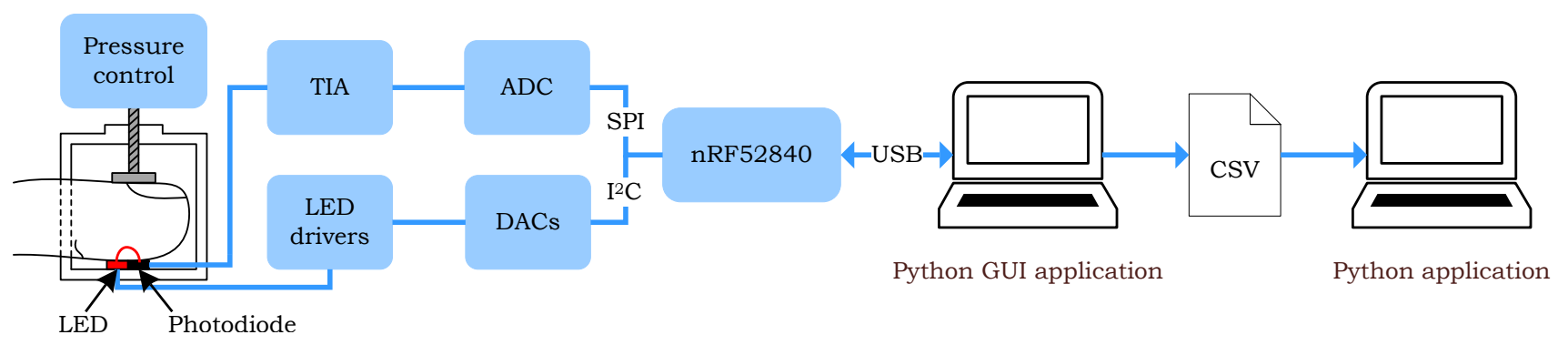

Figure 1. Block diagram of the system. An nRF52840 SoC controls two DACs and an ADC. The DACs together with the LED drivers control the LED currents and the turning of the LEDs on and off. The ADC digitises the voltage generated by the TIA circuit, which converts the photodiode current into voltage. The generated data is sent to a computer application, which visualises and saves the data. The saved data is analysed by a separate application.

a cable. This allows flexible placement of the sensor PCB, for example, to the fingertip or to the temple. The controlling PCB contains an LED driver for each of the five LEDs. The LED driving circuits are constant current sink circuits constructed from NPN bipolar junction transistors (BJT) in an emitter follower fashion. The constant current nature of the circuits guarantees stable LED light output characteristics. The current through the LEDs is controlled by two Microchip's 4-channel 12-bit MCP4728 digital-to-analog converters (DAC), which supply the base voltages of the transistors. Next to the LED drivers is a circuit for converting the current produced by the photodiode into voltage using a transimpedance amplifier (TIA). The produced voltage is fed into Analog Devices' 16-bit AD7680 successive approximation analog-to-digital converter (ADC). All of the components are powered from two ultralow noise low-dropout (LDO) linear regulators in order to improve the signal quality. The LED and the photodiode circuits are controlled by Nordic Semiconductor's nRF52840 system on a chip (SoC). The SoC was attached to the controlling PCB by soldering Nordic Semiconductor's nRF52840 Dongle, which is a small PCB containing the aforementioned SoC and a type A USB connector.

Performance-wise, the hardware is capable of achieving sampling rates of $625 \mathrm{~Hz}$ for all five channels and 1,000 $\mathrm{Hz}$ for three channels. High sampling rates improve the accuracy of locating the peaks and feet in a PPG signal.

\subsection{System Operation}

The firmware of the device was written in C programming language using the nRF5 software development kit (SDK). The firmware was built around timer interrupts to guarantee stable sampling rates: regular (the interval is defined by the desired sampling rate) and precise timer interrupts are triggered using a high frequency clock. Once an interrupt occurs, an ADC conversion is performed one after the other for each of the five channels together with unique timestamps. After creating the samples, an addi- tional ADC conversion is performed to record the level of ambient light.

The device is controlled by a developed Python-based computer application. The application connects to the device through a USB serial connection, which is used to send commands to the device and receive data. The application has a graphical user interface (GUI) allowing the user to quickly change some of the basic settings, such as the sampling rate and the current of the LEDs, but more importantly to monitor the signal quality from realtime graphs generated from the incoming data. The received data together with the device settings are stored as a comma-separated values (CSV) file.

\subsection{Algorithms}

The stored CSV file is analysed with a separate Python script. After parsing the file into NumPy arrays by the channel, the ambient light is subtracted from each corresponding sample. This is done to reduce possible distortions in the MWPPG signals caused by light artifacts. The signals are then inverted to make the pulse waveform familiar looking, i.e. the systolic peak marks the peak point in each cardiac cycle. The resulting signals are filtered with a band-pass filter to remove high frequency noise and to extract the pulsatile AC components, which are superimposed into large DC components. The type of the filter is a fourth-order Butterworth with a lower cutoff frequency at $0.5 \mathrm{~Hz}$ and higher cutoff frequency at $6.0 \mathrm{~Hz}$. The order of the filter is based on the results given in [8] and the cutoff frequencies were determined by analysing the power spectrum of a raw PPG signal obtained with fast Fourier transform (FFT).

The filtered PPG signals are then normalised and the pulse waveform peaks (i.e. the systolic peaks) and feet (i.e. the beginnings of systolic phases) are found with the automatic multiscale-based peak detection (AMPD) algorithm presented in [9]. The peaks and feet of each wavelength are then matched against the feet found in the infrared PPG 
A

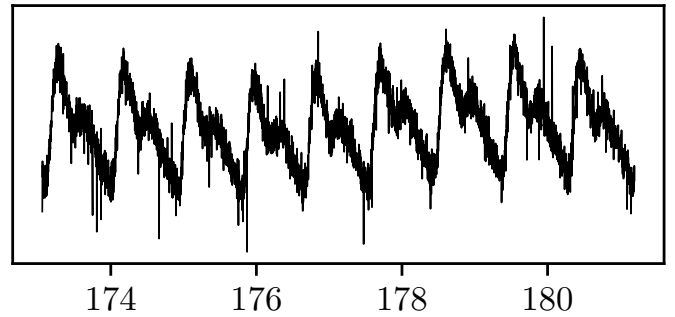

B

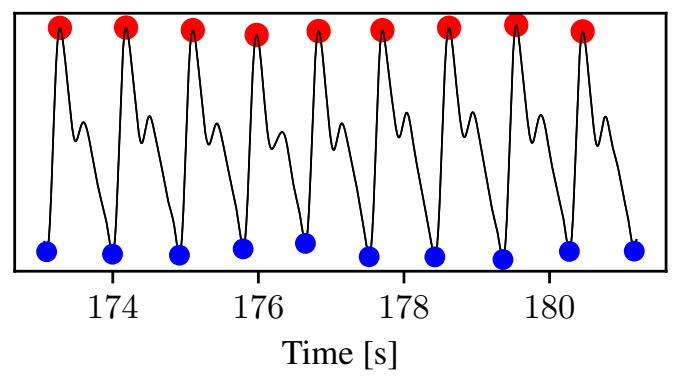

Figure 2. A) A sample of raw PPG signal measured at infrared wavelength. B) The same PPG signal filtered with feet (blue) and peaks (red) marked.

signal. Infrared was selected to be the reference signal because it penetrates deepest into the skin, and into the arteries, resulting in strong signal. The feet were selected instead of the peaks because they tend to be more stable than the peaks having been affected less by pressure wave reflections. In addition, the feet are a more natural choice than the peaks because they mark the opening of the aortic valve. Figure 1 shows a block diagram of the developed system. The external pressure control, shown in the figure, is performed manually with the help of a custom-designed small device.

\section{Results}

Figure 2 shows raw infrared PPG signal together with its filtered version where the feet and peaks have been marked with blue and red circles, respectively. The quality of the raw signal is good considering that it has not been filtered by any hardware filters and only the DC component has been subtracted from it in order to remove baseline wandering. Already from the raw signal it is possible to detect the systolic peaks, dicrotic notches and diastolic peaks. After filtering the pulse waveform becomes very clear, and the AMPD algorithm works well without any additional modifications. Part of the reason for the good signal quality is that the measurements were taken from a healthy individual at rest while in supine position. It is expected that the algorithm would not work as well if the measurement conditions were not as controlled.

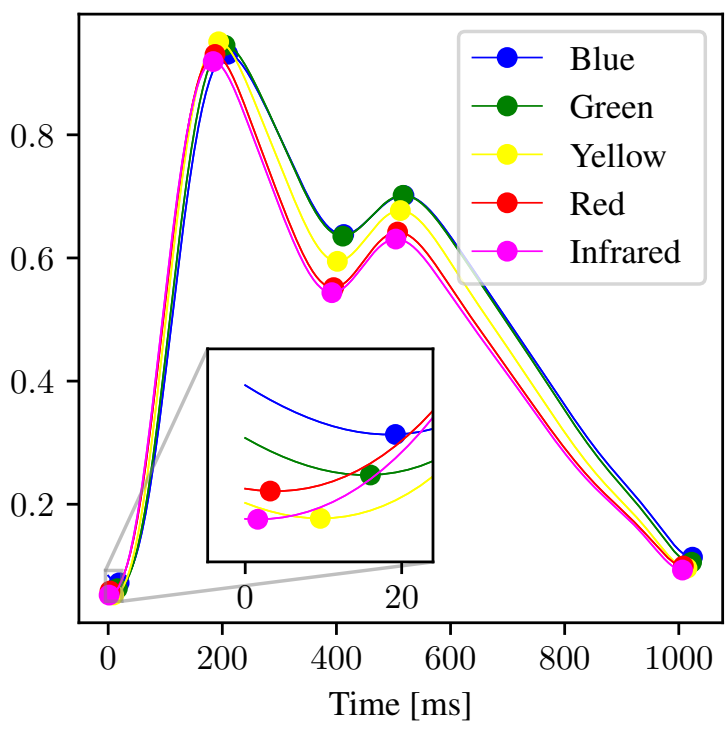

Figure 3. Average pulse waveforms computed at five different wavelengths over a measurement period of approximately three minutes. The waveforms have been matched against the infrared waveform feet. The beginnings of systolic phases, systolic peaks, dicrotic notches, diastolic peaks and diastolic ends have been marked with a filled circle.

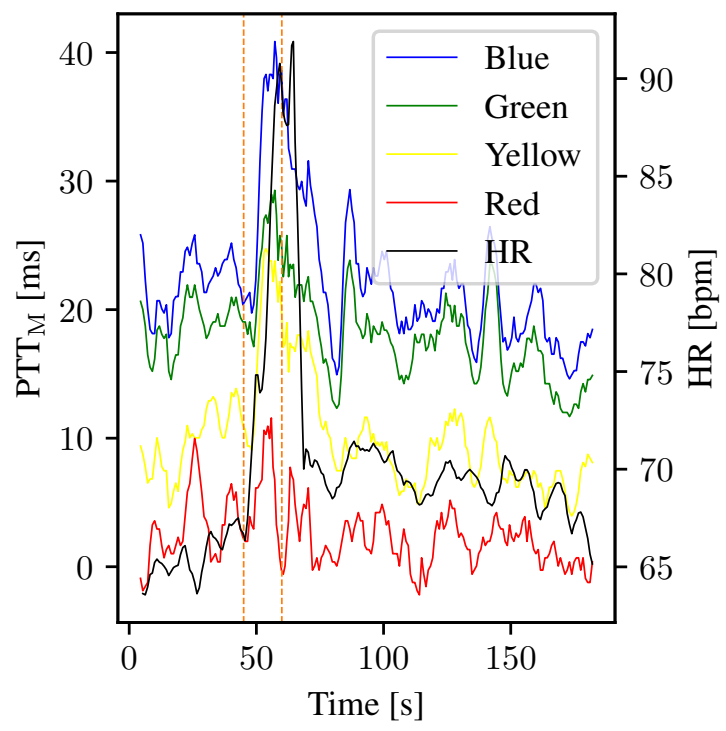

Figure 4. $\mathrm{PTT}_{\mathrm{M}}$ values calculated against infrared using the pulse waveform feet measured at blue, green, yellow and red wavelengths. The lines are 5-sample moving averages. A 15 -second Valsalva maneuver is marked by two orange vertical lines. 
Figure 3 shows the average pulse waveforms computed using each of the five wavelengths. By carefully observing the figure, it is possible to see that the feet (and the peaks) have a very small time difference, which is depended on the wavelength: the shorter the wavelength, the more delayed it is. This phenomenon can also be seen in the phase difference of the waveforms when observing the rising edges of the waveforms. These observations support the theory of how longer wavelength light penetrates deeper into the skin than shorter wavelength light.

$\mathrm{PTT}_{\mathrm{M}}$ values were calculated using the detected feet. Figure 4 shows the $\mathrm{PTT}_{\mathrm{M}}$ values for each channel calculated against the channel with the longest wavelength, i.e. infrared. Infrared was chosen to be the reference channel because as it is the longest wavelength, it is the best estimate of arterial pulsation. In addition, we are interested in computing the time it takes from the pulse wave to travel from the arteries to the arterioles and further to the capillaries. The figure shows that the $\mathrm{PTT}_{\mathrm{M}}$ values are in line with the assumption that longer wavelength light penetrates deeper into the skin: the delay is greatest at blue and green wavelengths, which are assumed to be proxies for the capillaries. The values are also in line with [4] where the $\mathrm{PTT}_{\mathrm{M}}$ values are described to be "about a few tens of milliseconds". During the measurement a Valsalva maneuver, i.e. expiring against closed glottis, was also performed to see how the maneuver affects the $\mathrm{PTT}_{\mathrm{M}}$. As figure 4 shows, the reaction is quite expected in that $\mathrm{PTT}_{\mathrm{M}}$ values increase due to the sudden decrease in systolic pressure caused by the maneuver. The simultaneously spiking heart rate (HR) proves that the maneuver was successful.

\section{Conclusions}

The developed device was able to produce good quality signal for computing $\mathrm{PTT}_{\mathrm{M}}$. Being able to study different blood vessels within the skin with a low-cost device offers interesting research topics in the area of hemodynamics. Future work will focus on studying further the nature of the computed $\mathrm{PTT}_{\mathrm{M}}$ values.

\section{Acknowledgements}

The work of Jukka-Pekka Sirkiä was funded by Business Finland (project ID: 2600455711).

\section{References}

[1] Laurent S, Cockcroft J, Van Bortel L, Boutouyrie P, Giannattasio C, Hayoz D, Pannier B, Vlachopoulos C, Wilkinson I, Struijker-Boudier H. Expert consensus document on arterial stiffness: methodological issues and clinical applications. European Heart Journal 09 2006;27(21):2588-2605. ISSN 0195-668X.
[2] Ma Y, Choi J, Hourlier-Fargette A, Xue Y, Chung HU, Lee JY, Wang X, Xie Z, Kang D, Wang H, Han S, Kang SK, Kang Y, Yu X, Slepian MJ, Raj MS, Model JB, Feng X, Ghaffari R, Rogers JA, Huang Y. Relation between blood pressure and pulse wave velocity for human arteries. Proceedings of the National Academy of Sciences 2018;115(44):11144-11149. ISSN 0027-8424.

[3] Liu J, Zhang Y, Ding X, Dai W, Zhao N. A preliminary study on multi-wavelength PPG based pulse transit time detection for cuffless blood pressure measurement. In 2016 38th Annual International Conference of the IEEE Engineering in Medicine and Biology Society (EMBC). 2016; 615-618.

[4] Liu J, Yan BP, Zhang Y, Ding X, Su P, Zhao N. Multi-wavelength photoplethysmography enabling continuous blood pressure measurement with compact wearable electronics. IEEE Transactions on Biomedical Engineering 2019;66(6):1514-1525.

[5] Yang M, Tuchin V, Yaroslavsky A. Principles of Light-Skin Interactions. Springer, 12 2009; 1-44.

[6] Bashkatov AN, Genina EA, Kochubey VI, Tuchin VV. Optical properties of human skin, subcutaneous and mucous tissues in the wavelength range from 400 to $2000 \mathrm{~nm}$. Journal of Physics D Applied Physics jul 2005;38(15):2543-2555.

[7] Panula T, Koivisto T, Pänkäälä M, Niiranen T, Kantola I, Kaisti M. An instrument for measuring blood pressure and assessing cardiovascular health from the fingertip. Biosensors and Bioelectronics 07 2020;112483.

[8] Liang Y, Elgendi M, Chen Z, Ward R. An optimal filter for short photoplethysmogram signals. Scientific Data May 2018;5(1):180076. ISSN 2052-4463.

[9] Scholkmann F, Boss J, Wolf M. An efficient algorithm for automatic peak detection in noisy periodic and quasiperiodic signals. Algorithms 2012;5(4):588-603. ISSN 1999-4893.

Address for correspondence:

Jukka-Pekka Sirkiä

Kiinamyllynkatu 10, 20520, Turku, Finland

jpsirk@utu.fi 